\title{
A Phase-Field Model for Liquid-Vapor Transitions
}

\author{
Alessia Berti` and Claudio Giorgi \\ Department of Mathematics, University of Brescia, via Valotti 9, \\ 25133 Brescia, Italy \\ ${ }^{\star}$ Corresponding author (alessia.berti@ing.unibs.it) \\ Communicated by W. Muschik, Berlin, Germany
}

\begin{abstract}
Starting from the mesoscopic description of the state equations for the vapor and liquid pure phases of a single chemical species, we propose a phase-field model ruling the liquid-vapor phase transition. Two different phases are separated by a thin layer, rather than a sharp interface, where the phase field changes abruptly from 0 to 1 . All thermodynamic quantities are allowed to vary inside the transition layer, including the mass density. The approach is based on an extra entropy flux which is proved to be non-vanishing inside the transition layer only. Unlike classical phase-field models, the kinetic equation for the phase variable is obtained as a consequence of thermodynamic restrictions and it depends only on the rescaled free enthalpy. The system turns out to be thermodynamically consistent and accounts for both temperature and pressure variations during the evaporation process. Few commonly accepted assumptions allow us to obtain the explicit expression of the Gibbs free enthalpy and the Clausius-Clapeyron formula. As a consequence, the customary form of the vapor pressure curve is recovered.
\end{abstract}

\section{Introduction}

Phase transitions occur in many relevant processes in physics and engineering and involve a large number of materials. In all of these processes, different phases are endowed with different structures or symmetries, hence we are allowed to introduce an order parameter, $\chi$, summarizing the structure order of the matter. According to a customary setting, the scalar parameter $\chi$, which characterizes the "amount of order" of the internal structure, takes its values in $\{0,1\}$, where $\chi=0$ corresponds to the less-ordered phase. An example occurs 
in a liquid-vapor phase transition, when a liquid phase vaporizes to a gaseous phase. In this particular case, the order parameter $\chi$ is identified with the liquid concentration, so that $\chi=0$ for the vapor and $\chi=1$ for the liquid. In the framework of a phase-field model, we propose here an oversimplified picture of non-isothermal and non-isobaric first-order transitions between liquid and vapor phases.

Let $\Omega$ be a fixed, bounded domain of $\mathbb{R}^{3}$ that is completely filled by one chemical component (water, for instance). We shall assume throughout that only two phases of the same particle species can occur. At the macroscopic scale, the domains occupied by the phases are separated by a sharp interface, and the order parameter is piecewise constant in $\Omega$. Since the domain evolution is not known a priori, and the interface between them evolves in time, this approach leads to a free-boundary moving problem where the main effort is to predict the localization and the evolution of the phase interfaces. This is also labeled as Stefan problem, and can be addressed from two (mathematically different) points of view: the classical formulation, where the dynamics is governed by the mean-curvature flow, and the weak formulation, where the problem may be reduced to a variational inequality (see [24]). Unfortunately, numerical simulations are very hard in this framework and no application examples are quantitatively comparable to the analytical model or experimental results.

In order to relax this computational difficulty, a thin interface limit approach, named phase-field modeling, has been recently adopted. The phase-field model for solidification was first formulated by Langer [14], then extended to different transition phenomena and analyzed mathematically and thermodynamically (see, for instance, $[1,3,4,10,20-22]$ and references therein). This point of view can be physically motivated by taking into account the finelength-scale effects, in that phase interfaces are regarded as thin layers, rather than sharp surfaces, where a mixture of the two phases occurs. This feature is captured if we introduce a regular scalar field $\varphi(\boldsymbol{x}, t)$, named phase field, whose values overlap the order parameter $\chi$ throughout the domain $\Omega$, except for a very thin region around the phase interfaces where $\nabla \varphi$ is noticeably different from zero. A specific contribution, which is named interface energy and depends on the width $\delta$ of that layer, must be added to the free energy expression. As proved in [13], phase-field models for first-order phase transitions are more tractable from the numerical point of view and in the limit $\delta \rightarrow 0$ they approach reasonably well free boundary problems for melting and nucleation processes [19].

Usually, non-isothermal transitions are depicted as temperature-induced phenomena that occur at constant pressure, say $p^{*}$ (see, for instance, $\left.[1,10,20]\right)$. In this case, the (absolute) temperature field $\theta(\boldsymbol{x}, t)$ must be added to the phase 
field in order to describe the evolution of the system. Then, a temperature value $\theta^{*}$, matching the fixed pressure $p^{*}$, is introduced into the model in such a way that the homogeneous stationary solutions $\varphi=0$ and $\varphi=1$ are both stable when $\theta=\theta^{*}$. This value is called transition temperature. In addition, it is usually assumed that the less-ordered phase $\varphi=0$ is stable when $\theta>\theta^{*}$ and, on the contrary, the phase $\varphi=1$ is stable when $\theta<\theta^{*}$. Such a behavior is justified by means of the special form of the free energy dependence on $\theta$ and $\varphi[7,8,13]$.

In order to take into account the effects due to the pressure variation, we need to introduce also the pressure field $p(\boldsymbol{x}, t)$. In general, a first-order phase transition occurs in $(\boldsymbol{x}, t)$ when $(\theta(\boldsymbol{x}, t), p(\boldsymbol{x}, t))$ crosses the transition point $\left(\theta^{*}, p^{*}\right)$. This is consistent with the observation that during phase changes both temperature and pressure keep constant values. Accounting for experimental data, for any specified pair of phases (i.e., solid-liquid, liquid-vapor, solid-vapor) this procedure yields a different set of points in the $(\theta, p)$ plane. Assembling these points, the resulting curves cross at the so-called triple point, $\left(\theta_{T}, p_{T}\right)$ and split the plane into three regions where each different phase is stable (see, e.g., [12]).

The goal of this paper is to construct a simple yet thermodynamically consistent phase-field model ruling the liquid-vapor phase transition and accounting for both temperature and pressure variations during the evaporation process. Our derivation takes care to introduce a slight set of (commonly accepted) assumptions and a clear thermodynamic framework. In addition, we take advantage of an explicit (oversimplified) representation of all the constitutive relations involved. To the best of our knowledge, most of the literature on phase transitions deals with processes at constant volume, although in experiments usually it is the pressure that is maintained constant. There are only few papers dealing with phase-field models allowing the system to change its density (see, e.g., $[7,17,20])$ and to undergo transitions jointly induced by temperature and pressure variations [6].

Plan of the paper. The construction of the model starts from the general approach to phase transitions devised in [7]. This approach, which is extensively summarized in Section 2, relies on a non-local form of the second law of thermodynamics and is characterized, as well as other phase-field theories (see, for instance, $[2,25]$ ), by the presence of an extra entropy flux $\boldsymbol{k}$. After exploiting the thermodynamic restrictions imposed by the second law, in Section 3 we are able to obtain the explicit expression of $\boldsymbol{k}$ and the general form of the kinetic equation for $\varphi$, which involves the rescaled free energy of the system only. This approach traces back to Maugin [15]. Section 4 is devoted to formulating the macroscopic constitutive equation for the pressure 
(equation of state) and the internal energy, taking into account that temperature and pressure are continuous, but the internal energy and mass density suffer a jump across the phase-change region. In particular, we look for a global description that is able to smoothly connect these extensive quantities across the interface layer. The complete expression of the Helmholtz free energy and Gibbs free enthalpy densities is then derived in Section 5 by means of the standard exploitation of thermodynamic restrictions and constitutive assumptions. In Section 6, the Clausius-Clapeyron formula, jointly with the customary form of the vapor pressure curve, are recovered. Finally, in Section 7, we write explicitly the governing equations of the system and their linearization in a neighborhood of a point of the vapor-pressure curve. Conclusions are addressed in Section 8, and Section 9 collects the proofs of propositions occurring in the paper.

\section{Thermodynamics of non-local phase-field models}

The liquid-vapor system is assumed to occupy a fixed bounded domain $\Omega \subset$ $\mathbb{R}^{3}$ with smooth boundary $\partial \Omega$, whose unit outward normal is denoted by $\boldsymbol{n}$. Let $t$ be the time variable, $\boldsymbol{x}, \boldsymbol{v}$ the position vector and the velocity of the particle at time $t$ in the actual configuration, $\theta$ the absolute temperature, $\rho$ the mass density. We denote by $\operatorname{sym} \boldsymbol{A}, \operatorname{skw} \boldsymbol{A}$, respectively, the symmetrical and skew part of a tensor $\boldsymbol{A}$. Moreover, we denote by $\boldsymbol{A}^{\circ}$ the deviatoric (or traceless) part of a symmetric tensor. In particular, we define

$$
\boldsymbol{L}=\nabla \boldsymbol{v}=\boldsymbol{D}+\boldsymbol{\Omega}, \quad \boldsymbol{D}=\operatorname{sym} \boldsymbol{L}, \quad \boldsymbol{\Omega}=\operatorname{skw} \boldsymbol{L},
$$

where $\nabla=\partial_{x}$ is the gradient operator. Moreover, we use the superposed dot to denote the total time derivative, namely $\dot{f}(\boldsymbol{x}, t)=\partial_{t} f(\boldsymbol{x}, t)+\boldsymbol{v}(\boldsymbol{x}, t) \cdot \nabla f(\boldsymbol{x}, t)$. We start by writing the balance equations of mass, linear momentum, and energy in the eulerian form

$$
\begin{aligned}
& \dot{\rho}+\rho \nabla \cdot \boldsymbol{v}=0, \\
& \rho \dot{\boldsymbol{v}}=\nabla \cdot \boldsymbol{T}+\rho \boldsymbol{b}, \\
& \rho \dot{\varepsilon}=\boldsymbol{T} \cdot \boldsymbol{D}-\nabla \cdot \boldsymbol{q}+\rho r,
\end{aligned}
$$

where $\boldsymbol{T}$ is the Cauchy stress tensor, $\boldsymbol{b}$ the body force density, $\varepsilon$ the internal energy density, $\boldsymbol{q}$ the heat flux vector, and $r$ the external heat supply density. From Eg. (2) 1 we deduce the equality

$$
\boldsymbol{D} \cdot \boldsymbol{I}=\nabla \cdot \boldsymbol{v}=-\frac{\dot{\rho}}{\rho} .
$$


In order to take viscous fluids into account, we suppose that $\boldsymbol{T}$ satisfies the constitutive equation

$$
\boldsymbol{T}=-p \boldsymbol{I}+\boldsymbol{S},
$$

where $p$ denotes the pressure, $\boldsymbol{I}$ stands for the identity tensor, and $\boldsymbol{S}$ is a symmetrical tensor.

Since our goal is to describe a transition between two phases (vapor and liquid) of the same particle species, we introduce a model where a single scalar function

$$
\varphi(\boldsymbol{x}, t): \Omega \times[0, T] \rightarrow[0,1],
$$

called phase-field, is involved. More precisely, we attach to each particle the auxiliary function $\varphi$ such that $\varphi=0$ represents the less ordered phase (vapor) while $\varphi=1$ characterizes the most ordered one (liquid). It is worth noting that $\varphi$ is not necessarily coincident with the concentration of the liquid as in a scheme of fluid mixtures (see [16]). Following the phase-field approach, as outlined in [22], we suppose that the two pure phases are separated by thin transition layers with finite thickness, called diffuse interfaces, and we assume that $\varphi \in[0,1]$ varies smoothly across these layers.

Moreover, the mass density $\rho$ and the velocity $v$ coincide with the density and the velocity of the vapor (or the liquid) in the regions where only vapor (or liquid) appears, whereas they are suitable, but a priori unknown, superpositions of the densities and the velocities of the vapor and of the liquid in the diffuse interface.

As is well known, at the macroscopic level a transition layer looks like a sharp interface whose motion is governed by a mean-curvature flow equation. Because of this feature, phase transitions are customarily considered as nonlocal phenomena (see [24]). In a mesoscopic model, non-local spatial effects can be taken into account by assuming that the quantities involved in the constitutive equations depend on the values of $\rho, \theta, \varphi, \boldsymbol{D}^{\circ}$ in $\boldsymbol{x}$ and on their gradients up to some order greater than 1 . Here, for the sake of simplicity, we consider the set

$$
\Sigma=\left(\rho, \theta, \varphi, \boldsymbol{D}^{\circ}, \nabla \rho, \nabla \theta, \nabla \varphi, \nabla \boldsymbol{D}^{\circ}, \nabla \nabla \rho, \nabla \nabla \theta, \nabla \nabla \varphi\right)
$$

and we neglect third- and higher-order gradients. Furthermore, we suppose that there exists a suitable function $\Phi$ such that the evolution of $\varphi$ is described by the equation

$$
\dot{\varphi}=\Phi(\Sigma) .
$$


Also we assume that the dependence of $\Phi$ on $\nabla \varphi$ is non-trivial. As is apparent, this formulation is general enough to include the most common non-conserved scalar phase-field theories based on the Ginzburg-Landau pioneering work (see [20]). Since the choice of $\Phi$ as a function of $\Sigma$ is submitted to restrictions mainly due to the second law of thermodynamics (see [21]), we first give a statement for this law that is compatible with the non-local character of the phenomena involved.

Let $\eta$ be the entropy density, $\boldsymbol{Q}$ the entropy flux, and $\sigma$ the entropy supply density. We express the second law of thermodynamics through the following statement:

Entropy principle. For all fields $\rho, \theta, \boldsymbol{v}, \boldsymbol{T}, \boldsymbol{q}, \boldsymbol{b}, r$ compatible with the balance laws (2), the following equality holds:

$$
\frac{d}{d t} \int_{\Omega} \rho \eta d v=-\int_{\partial \Omega} \boldsymbol{Q} \cdot \boldsymbol{n} d a+\int_{\Omega} \rho \sigma d v
$$

where $\sigma=\sigma_{1}+\sigma_{2}$ and $\sigma_{1}, \sigma_{2}$ satisfy the conditions

$$
\sigma_{1}=\frac{r}{\theta}, \quad \sigma_{2} \geq 0, \quad \text { in } \Omega \times \mathbb{R}^{+} .
$$

We stress that Eq. (6) is assumed on the whole domain $\Omega$ only, thus this statement of the second law has a non-local nature (see $[11,18]$ ). If now we assume, as usual, the entropy flux $\boldsymbol{Q}$ in the form

$$
\boldsymbol{Q}=\frac{\boldsymbol{q}}{\theta},
$$

then, by applying the divergence theorem, from Eq. (6) we obtain

$$
\int_{\Omega} \rho \dot{\eta} d v=-\int_{\Omega} \nabla \cdot\left(\frac{\boldsymbol{q}}{\theta}\right) d v+\int_{\Omega} \frac{\rho r}{\theta} d v+\int_{\Omega} \rho \sigma_{2} d v
$$

This is equivalent to stating that

$$
\rho \dot{\eta}+\nabla \cdot\left(\frac{\boldsymbol{q}}{\theta}\right)-\frac{\rho r}{\theta}-\rho \sigma_{2}=-\nabla \cdot \boldsymbol{k},
$$

where $\boldsymbol{k}=\boldsymbol{k}(\boldsymbol{x}, t)$ is a regular field, called extra entropy flux, such that

$$
\int_{\Omega} \nabla \cdot \boldsymbol{k} d v=\int_{\partial \Omega} \boldsymbol{k} \cdot \boldsymbol{n} d a=0
$$

As a consequence, inside $\Omega$ the entropy inequality (6) takes the form

$$
\rho \dot{\eta} \geq-\nabla \cdot\left(\frac{\boldsymbol{q}}{\theta}+\boldsymbol{k}\right)+\frac{\rho r}{\theta},
$$


where the entropy flux vector is redefined up to the extra contribution $\boldsymbol{k}$, namely

$$
Q^{\prime}=\frac{q}{\theta}+\boldsymbol{k}
$$

It is worth noting that

$$
\int_{\partial \Omega} \boldsymbol{Q}^{\prime} \cdot \boldsymbol{n} d a=\int_{\partial \boldsymbol{\Omega}} \boldsymbol{Q} \cdot \boldsymbol{n} d a=\int_{\partial \Omega} \frac{\boldsymbol{q}}{\theta} \cdot \boldsymbol{n} d a .
$$

\section{Thermodynamic restrictions}

This section concerns the thermodynamic restrictions imposed on the quantities $\eta, \boldsymbol{T}, \Phi, \boldsymbol{q}, \boldsymbol{k}$. We denote by $\psi=\psi(\Sigma)$ the Helmholtz free energy density. By differentiating relation $\psi=\varepsilon-\eta \theta$ with respect to $t$ and substituting Eqs. (2) $)_{3}$ and (8), we obtain the Clausius-Duhem inequality

$$
-\rho(\dot{\psi}+\eta \dot{\theta})+\boldsymbol{T} \cdot \boldsymbol{D}-\frac{1}{\theta} \boldsymbol{q} \cdot \nabla \theta+\theta \nabla \cdot \boldsymbol{k} \geq 0 .
$$

As customary (see $[1,7])$, we assume the following statement:

Assumption 3.1 The entropy extra flux $\boldsymbol{k}$ is homogeneous with respect to $\dot{\varphi}$. In other words, if no phase change occurs $(\dot{\varphi}=0)$, then $\boldsymbol{k}$ vanishes.

Hence, as shown in the Appendix, the following result holds:

Proposition 3.2 The functions $\psi, \eta, \boldsymbol{T}, \Phi, \boldsymbol{q}, \boldsymbol{k}$ are compatible with the second law of thermodynamics if

$$
\begin{array}{rlrl}
\psi & =\psi(\rho, \theta, \varphi, \nabla \varphi), & & \operatorname{skw}(\nabla \varphi \otimes \partial \nabla \varphi \psi)=\mathbf{0}, \\
p & =\rho^{2} \partial_{\rho} \psi, & \eta=-\partial_{\theta} \psi, \\
\boldsymbol{k} & =\frac{1}{\theta} \rho \partial_{\nabla \varphi} \psi \dot{\varphi} &
\end{array}
$$

and Clausius-Duhem inequality reduces to

$$
\begin{aligned}
\frac{1}{\theta^{2}} \boldsymbol{q} \cdot \nabla \theta+\left[\partial_{\varphi}\left(\frac{\rho \psi}{\theta}\right)-\nabla\right. & \left.\cdot \partial \nabla \varphi\left(\frac{\rho \psi}{\theta}\right)\right] \Phi \\
& -\frac{1}{\theta}(\boldsymbol{S}+\rho \nabla \varphi \otimes \partial \nabla \varphi \psi) \cdot \boldsymbol{D} \leq 0 .
\end{aligned}
$$


It is apparent from Eq. $(10)_{3}$ that the entropy extra flux is non-zero only in the interface layer where $\nabla \varphi \neq \mathbf{0}$. This means that in the pure phases the Clausius-Duhem inequality reduces to the standard form [5]

$$
-\rho(\dot{\psi}+\eta \dot{\theta})+\boldsymbol{T} \cdot \boldsymbol{D}-\frac{1}{\theta} \boldsymbol{q} \cdot \nabla \theta \geq 0 .
$$

Notice that in a scheme of binary mixtures, the entropy extra flux is proportional to the relative velocity between both constituents. This is not the point of view of this paper, where phases are not considered as two distinct fluids. In modeling phase transitions where mixtures are involved, two terms occur in the entropy extra flux, as pointed out in [16].

In order to satisfy Eq. (11), it is natural to assume that

$$
\begin{aligned}
\boldsymbol{q}=-\kappa \nabla \theta, \quad \Phi=-\lambda\left[\partial_{\varphi}\left(\frac{\rho \psi}{\theta}\right)-\nabla \cdot \partial_{\nabla \varphi}\left(\frac{\rho \psi}{\theta}\right)\right] & , \\
\boldsymbol{S} & =\chi \boldsymbol{D}-\rho \nabla \varphi \otimes \partial \nabla \varphi \psi,
\end{aligned}
$$

where $\kappa, \lambda, \chi$ are positive functions depending on $\Sigma$.

Remark 3.3 Equations (5) and (12) 2 rule the evolution of $\varphi$, which means that the transition depends only on the rescaled Helmholtz free energy density $\psi / \theta$ (see $[1,21]$ ). We denote by $\delta_{\varphi}$ the variational derivative with respect to $\varphi$, i.e.,

$$
\delta_{\varphi} \Xi=\partial_{\varphi} \Xi-\nabla \cdot \partial_{\nabla \varphi} \Xi
$$

for every scalar function $\Xi$. Then, Eqs. (5) and (12) 2 yield

$$
\dot{\varphi}=-\lambda \delta_{\varphi}\left(\frac{\rho \psi}{\theta}\right)=-\lambda \delta_{\varphi}\left(\frac{\bar{F}}{\theta}\right),
$$

where $\bar{F}=\rho \psi$ is the Helmholtz free energy function. It follows from the previous relation that the stationary points of $\bar{F} / \theta$ satisfies $\dot{\varphi}=0$, in agreement with the point of view of [1], where the critical points of the rescaled free energy are the solutions of the stationary problem (see also [3]).

\section{Pressure and internal energy}

In this section, we give the constitutive equations for the pressure $p$ and the internal energy density $\varepsilon$ in the transition layer. To this aim, we proceed in two steps. First, we choose constitutive equations for $p$ and $\varepsilon$ in the two pure phases, 
then we extend them to the whole domain $\Omega$ by means of a smooth function of the order parameter $\varphi$. The choice is performed in such a way that $\theta$ and $p$ keep constant values (and hence are continuous) across the transition layer, while $\rho$ and $\varepsilon$ suffer a jump discontinuity. In addition, constitutive relations are chosen as simply as possible since we are interested in the qualitative behavior of the resulting transition model only. In the next section it will be clear that if we give the constitutive equations for the pressure $p$ and the internal energy $\varepsilon$, then we can recover (up to a constant) the complete expression of the Helmholtz free energy. It is worth noting that usually a different (but equivalent) approach is adopted. Indeed, often one gives a thermodynamically consistent expression of $\psi$ and later deduce the constitutive equations for $p$ and $\varepsilon$.

\subsection{The energy-temperature diagram}

As is well known, both in the vapor and in the liquid phase the internal energy density $\varepsilon$ is a function of the temperature $\theta$ only. Letting $\varepsilon_{0}$ be the internal energy in the vapor and $\varepsilon_{1}$ in the liquid, it is customary to assume that

$$
\varepsilon_{0}(\theta)=c_{0} \theta, \quad \varepsilon_{1}(\theta)=c_{1} \theta-L,
$$

where $L$ is constant and strictly positive and the rates $c_{0}$ and $c_{1}$ denote the vapor- and liquid-specific heat at constant volume, respectively. When water is concerned, the specific heat of the vapor is smaller than that of the liquid, and $c_{0}<c_{1}$, accordingly. The positive jump of the energy at the critical temperature $\theta^{*}$, namely

$$
\varepsilon_{0}\left(\theta^{*}\right)-\varepsilon_{1}\left(\theta^{*}\right)=\left[c_{0}-c_{1}\right] \theta^{*}+L,
$$

represents the latent heat $L_{v}$ absorbed during the evaporation process and is related to the length of the horizontal segment shown in Figure 1 (on the left the liquid energy, $\theta<\theta^{*}$, on the right the vapor energy, $\theta>\theta^{*}$ ). If $c_{0}=c_{1}$, then $L_{v}=L$.

\subsection{The Andrews density-pressure diagrams}

Now, our aim is to exploit the similarity between the temperature-energy diagram and the Andrews diagram (see [23]) in order to represent both in the liquid and vapor phases the equations of state that involve pressure, temperature, and mass density. The Andrews diagram is obtained by applying the Maxwell construction to the isotherms of the van der Waals equation (see, for instance, [12], p. 67). It mainly differs from the energy-temperature diagram by the presence of three variables rather than two. In fact, in the density- 


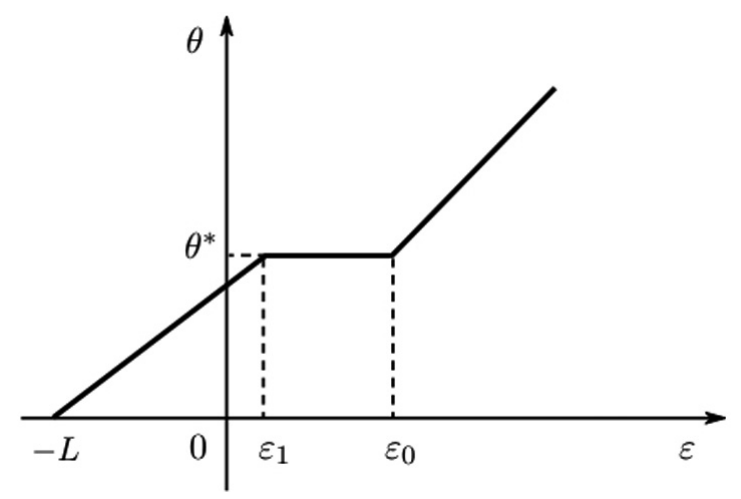

Figure 1 The energy-temperature diagram.

pressure plane we have a family of curves depending on the parameter $\theta$ (isothermal curves).

Let $\rho_{0}$ and $\rho_{1}$ be the density of the vapor and the liquid, respectively. We denote by $v_{0}, v_{1}$ the corresponding specific volumes, i.e., $v_{0}=1 / \rho_{0}$ and $v_{1}=1 / \rho_{1}$. As observed in the previous subsection, when $\theta \ll \theta_{c}$ then $\rho_{0} \ll \rho_{1}$ and $v_{0} \gg v_{1}$. It is quite reasonable to suppose that the vapor behaves like a perfect gas, so that

$$
p_{0} v_{0}=k \theta \text {. }
$$

This equation looks like a constitutive (state) equation for $p_{0}$ at fixed temperature, namely

$$
p_{0}=k \theta \rho_{0} .
$$

Accordingly, we assume a similar constitutive law in the liquid phase,

$$
p_{1}=k \theta[1-\hat{v}(\theta)] \rho_{1},
$$

where $\hat{v}$ is a decreasing function of $\theta$ such that $\hat{v}(\theta) \in[0,1]$ and satisfies the condition

$$
\hat{v}(\theta)=0, \quad \theta \geq \theta_{c},
$$

where $\theta_{c}$ is the critical temperature. Indeed, when $\theta \geq \theta_{c}$ the vapor cannot be liquefied at any pressure and hence the constitutive equation (14) should reduce to Eq. (15). For instance, we may choose

$$
\hat{v}(\theta)= \begin{cases}\left(1-\frac{\theta}{\theta_{c}}\right) v & \text { if } 0<\theta<\theta_{c}, \\ 0 & \text { if } \theta \geq \theta_{c},\end{cases}
$$


where $0<v<1$, so that Eq. (15) takes the form

$$
p_{1}= \begin{cases}\frac{k}{\theta_{c}} \theta^{2} \rho_{1} & \theta<\theta_{c}, \\ k \theta \rho_{1} & \theta \geq \theta_{c} .\end{cases}
$$

The critical value $v_{c}$ can be obtained from $\theta_{c}$ by virtue of the relation

$$
v_{c}=\frac{k \theta_{c}}{p_{c}}=\frac{k \theta_{c}}{\pi\left(\theta_{c}\right)}
$$

where $\pi$ is the vapor pressure curve, i.e., the curve between the regions of vapor and liquid stability in the $(\theta, p)$ plane (see [12]).

We are finally in a position to evaluate the jump of density occurring at the phase change. To this end, we remember that both temperature and pressure keep constant across the transition process. Thus, we consider the isothermal curve $\theta=\theta^{*}$ in the Andrews diagram and, accounting for the knowledge of the vapor pressure function $\pi_{v}$, we are able to compute the value $p^{*}=\pi\left(\theta^{*}\right)$ at which the transition starts. During the evaporation process, the specific volume $v$ (and hence the mass density $\rho$ ) moves from its value $v_{1}\left(\rho_{1}\right)$ in the liquid phase to the corresponding value $v_{0}\left(\rho_{0}\right)$ in the vapor phase, and both depend on $\theta^{*}$, i.e.,

$$
\begin{aligned}
& v_{0}\left(\theta^{*}\right)=\frac{1}{\rho_{0}\left(\theta^{*}\right)}=\frac{k \theta^{*}}{\pi\left(\theta^{*}\right)}, \\
& v_{1}\left(\theta^{*}\right)=\frac{1}{\rho_{1}\left(\theta^{*}\right)}=\frac{k \theta^{*}\left[1-\hat{v}\left(\theta^{*}\right)\right]}{\pi\left(\theta^{*}\right)} .
\end{aligned}
$$

The assumption $\hat{v}\left(\theta^{*}\right) \in[0,1)$ guarantees that $v_{1}\left(\theta^{*}\right)<v_{0}\left(\theta^{*}\right)$. Hence the positive jump of the specific volume during the transition is given by

$$
v_{0}\left(\theta^{*}\right)-v_{1}\left(\theta^{*}\right)=\frac{k \hat{v}\left(\theta^{*}\right) \theta^{*}}{\pi\left(\theta^{*}\right)} .
$$

This quantity decreases when the temperature increases and vanishes as $\theta \rightarrow$ $\theta_{c}$ (see Figure 2).

\subsection{Constitutive equations for $p$ and $\varepsilon$}

Since we represent the phase change region as a thin layer rather than a sharp interface, we have to connect the pure phase constitutive equations for $\varepsilon_{0}, \varepsilon_{1}$, 


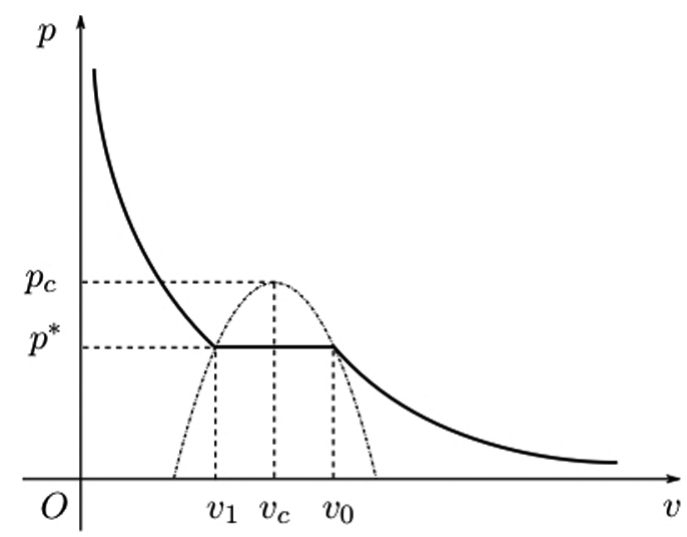

Figure 2 The density-pressure diagram.

and $p_{0}, p_{1}$ (as devised in previous subsections) inside the layer. To this aim, we introduce two functions of the phase-field $\varphi$ in such a way that $\theta$ and $p$ keep constant (and hence continuous) across the transition layer, whereas $\varepsilon$ and $\rho$ suffer a jump discontinuity. More precisely, we make the following

Assumption 4.1 The pressure and internal energy satisfies the constitutive equations

$$
\begin{aligned}
p(\rho, \theta, \varphi) & =k \rho \theta[1-v g(\varphi)], \\
\varepsilon(\theta, \varphi) & =c(\varphi) \theta+L f(\varphi)
\end{aligned}
$$

with $f, g$ smooth functions satisfying the conditions

$$
g(0)=f(0)=0, \quad g(1)=-f(1)=1 .
$$

As expected, Eqs. (13), (14), and (15) follow from the evaluation of Eqs. (17) and (18) at $\varphi=0,1$.

Taking advantage of Assumption 4.1, we recover the expression of $\psi$ thanks to the constitutive equations

$$
p=\rho^{2} \partial_{\rho} \psi, \quad \varepsilon=\psi-\theta \partial_{\theta} \psi .
$$

Certainly, the assumption that both vapor and liquid obey the constitutive equation of perfect gases and the linearity of $\varepsilon$ with respect to $\theta$ are somewhat restrictive. However, our purpose consists in exhibiting a simple model that is consistent with the Clausius-Duhem inequality and, at the same time, accounts for the usual picture of energy-temperature and density-pressure 
diagrams of phase transitions that are represented in any textbook dealing with thermodynamics.

Remark 4.2 A lot of different expressions for $f$ and $g$ can be found in the literature (see for instance, $[3,13,20]$ ). Mostly, $g$ is assumed to be odd (linear or cubic), whereas the growth of $f$ is even (typically of the fourth order).

Here and in the following, we suppose that $\theta \ll \theta_{c}$ and, for the sake of simplicity, we let $\hat{v}=v$ be constant. Moreover, since $v_{1} \ll v_{0}$, we infer that $v$ lies between $1 / 2$ and 1 . Furthermore, we let

$$
c(\varphi)=c_{P}-k[1-v g(\varphi)]=c_{V}+k v g(\varphi),
$$

where $c_{V}$ and $c_{P}=c_{V}+k$ are the specific heat at constant volume and pressure in the vapor phase. Then, we have

$$
c_{0}=c(0)=c_{V}, \quad c_{1}=c(1)=c_{V}+k v,
$$

and, according to previous statements, $c_{0}<c_{1}$.

Remark 4.3 Since during the transition both pressure and temperature keep constant values, $(p, \theta)=\left(p^{*}, \theta^{*}\right)$, the specific volume $v$ in the transition layer depends on the order parameter $\varphi$, only. In particular,

$$
v(\varphi)=v_{1} g(\varphi)+v_{0}[1-g(\varphi)],
$$

which generalizes the usual linear relation $v(\varphi)=v_{1} \varphi+v_{0}(1-\varphi)$.

Proof. After substituting $\theta=\theta^{*}$ and $p=p^{*}$ into Eq. (17) we obtain

$$
v(\varphi)=\frac{1}{\rho(\varphi)}=\frac{k \theta^{*}}{p^{*}}[1-v g(\varphi)]=\frac{k \theta^{*}}{p^{*}}(1-v) g(\varphi)+\frac{k \theta^{*}}{p^{*}}[1-g(\varphi)],
$$

and accounting for Eq. (16), we have the required expression of the specific volume.

Formula (22), which in our model is a consequence of constitutive equations, can be considered as the starting point for deriving the shape of $p$ and $\varepsilon$, according to a physical/chemical point of view.

\section{Thermodynamic potentials}

This section is devoted to determining an explicit expression of the Helmholtz free energy density $\psi$, which is able to describe the phase transition induced 
both by the temperature and by the pressure, according to constitutive equations (17) and (18).

Thermodynamic restrictions imply that $\psi$ depends only on $\rho, \theta, \varphi, \nabla \varphi$. Here we specify the expression of the free energy by supposing

$$
\psi(\rho, \theta, \varphi, \nabla \varphi)=\Psi(\rho, \theta, \varphi)+\hat{\psi}(\theta, \varphi)+\frac{1}{2} \mu(\theta)|\nabla \varphi|^{2} .
$$

The last term accounts for the interface energy between the two pure phases and it is in agreement with thermodynamic restriction $(10)_{1}$. The function $\mu$ is assumed to be positive valued since, as is well known (see [3]), $\psi$ must attain a minimum at the homogeneous phases, i.e., $\nabla \varphi=\mathbf{0}$. In particular, when $\mu$ is constant, we recover the customary quadratic dependence on $\nabla \varphi$ introduced by Cahn-Hilliard (see [3]). The first term represents the stored mechanical energy and in the present case its expression involves the pressure function. The second addendum is merely introduced to account for the thermodynamic condition

$$
\varepsilon=\psi+\eta \theta
$$

where $\varepsilon$ and $\eta$ are given by Eqs. (18) and (10)2, respectively.

From Eq. (18) it is apparent that the internal energy $\varepsilon$ is independent of $|\nabla \varphi|$. Thus, by substituting Eq. (23) with Eq. (20) 2 , we deduce that the function $\mu$ has to satisfy

$$
\mu^{\prime}(\theta) \theta=\mu(\theta)
$$

which implies

$$
\mu(\theta)=\mu_{0} \theta, \quad \mu_{0}>0 .
$$

The constant $\mu_{0}$ is positive valued since $\mu$ is a positive function.

Let us determine $\Psi$. Thermodynamic restrictions $(10)_{2}$, expression (23), and constitutive equation (17) lead to the equalities

$$
\partial_{\rho} \Psi=\partial_{\rho} \psi=\frac{p}{\rho^{2}}=\frac{k \theta[1-v g(\varphi)]}{\rho} .
$$

Therefore,

$$
\Psi(\rho, \theta, \varphi)=k \theta[1-v g(\varphi)] \ln \rho+h(\theta, \varphi),
$$

where $h$ is a generic function depending only on $\theta$ and $\varphi$. We introduce the pressure function $\mathcal{P}$ defined as

$$
\mathcal{P}=\int_{p_{c}}^{p} \frac{1}{\rho(\xi)} d \xi
$$


where $p_{c}$ is a constant reference value whose physical meaning will be explained in the following. The constitutive equation (17) yields

$$
\mathcal{P}(p, \theta, \varphi)=k \theta[1-v g(\varphi)] \ln \frac{p}{p_{c}} .
$$

We choose the function $h(\theta, \varphi)$ such that $\Psi=\mathcal{P}$. That is possible, in view of Eq. (17), by letting

$$
h(\theta, \varphi)=k \theta[1-v g(\varphi)] \ln \frac{k \theta[1-v g(\varphi)]}{p_{c}} .
$$

By substituting $\Psi=\mathcal{P}$ into Eq. (23), we obtain

$$
\begin{aligned}
& \psi(\rho, \theta, \varphi, \nabla \varphi)= \\
& \hat{\psi}(\theta, \varphi)+k \theta[1-v g(\varphi)] \ln \frac{p(\rho, \theta, \varphi)}{p_{c}}+\frac{1}{2} \mu_{0} \theta|\nabla \varphi|^{2},
\end{aligned}
$$

where $p$ satisfies Eq. (17).

Now our aim consists in determining $\hat{\psi}$. Substitution of Eqs. (18) and (24) into Eq. $(20)_{2}$ leads to the differential equation

$$
\hat{\psi}=c(\varphi) \theta+L f(\varphi)+\theta \partial_{\theta} \hat{\psi}+k \theta[1-v g(\varphi)] .
$$

In view of Eq. (21), we deduce that

$$
\theta \partial_{\theta} \hat{\psi}=\hat{\psi}-c_{P} \theta-L f(\varphi) \text {. }
$$

Among all the solutions of Eq. (25), we choose

$$
\hat{\psi}=c(\varphi) \theta-c_{P} \theta \ln \theta+L f(\varphi)=c_{P} \theta(1-\ln \theta)-k \theta[1-v g(\varphi)]+L f(\varphi) .
$$

As a consequence of the previous arguments, the Helmholtz free energy density $\psi$ is given by

$$
\begin{aligned}
\psi(\rho, \theta, \varphi, \nabla \varphi)= & c_{P} \theta(1-\ln \theta)+L f(\varphi)+k \theta[1-v g(\varphi)] \\
& \left(\ln \frac{\rho k \theta[1-v g(\varphi)]}{p_{c}}-1\right)+\frac{1}{2} \mu_{0} \theta|\nabla \varphi|^{2}
\end{aligned}
$$

In particular, on account of conditions (19) and equality $c_{P}=c_{V}-k$, we have

$$
\psi(\rho, \theta, 0, \mathbf{0})=c_{V} \theta(1-\ln \theta)+k \theta\left(\ln \rho+\ln \frac{k}{p_{c}}\right),
$$

which coincides with [9, formula (119)] for an ideal gas, up to a constant addendum. 
Similarly, the Helmholtz free energy density of the liquid in a uniform configuration is given by

$$
\psi(\rho, \theta, 1, \mathbf{0})=c_{P} \theta(1-\ln \theta)-L+k \theta(1-v)\left(\ln \frac{\rho k \theta(1-v)}{p_{c}}-1\right) .
$$

Since the temperature and the pressure keep constant during the transition, we look for the expression of some energy potentials that naturally depends on $p$ and $\theta$, rather than $\rho$ and $\theta$. To this aim, we introduce the Gibbs free enthalpy $\Gamma$, defined as

$$
\Gamma=N \psi+p V
$$

where $N$ denotes the particle number and $V=N / \rho$ is the volume. Its density $\gamma$, also called chemical potential, is defined through the equality

$$
\gamma=\frac{\Gamma}{N}=\psi+\frac{p}{\rho} .
$$

In view of Eqs. (17) and (26), the density $\gamma$ reads

$$
\begin{aligned}
\gamma(\rho, \theta, \varphi, \nabla \varphi)= & c_{P} \theta(1-\ln \theta)+L f(\varphi)+k \theta[1-v g(\varphi)] \\
& \ln \frac{\rho k \theta[1-v g(\varphi)]}{p_{c}}+\frac{1}{2} \mu_{0} \theta|\nabla \varphi|^{2} .
\end{aligned}
$$

For later convenience, we define $\tilde{\gamma}=\gamma(\rho(p, \theta, \varphi), \theta, \varphi, \nabla \varphi)$ so that

$$
\begin{aligned}
\tilde{\gamma}(p, \theta, \varphi, \nabla \varphi)= & c_{P} \theta(1-\ln \theta)+L f(\varphi)+k \theta[1-\nu g(\varphi)] \\
& \ln \frac{p}{p_{c}}+\frac{1}{2} \mu_{0} \theta|\nabla \varphi|^{2} .
\end{aligned}
$$

In the next section, we are going to use $\tilde{\gamma}$ to deduce the Clausius-Clapeyron equation.

\section{Uniform phase changes: Clausius-Clapeyron equation}

In this section, we are able to state the Clausius-Clapeyron equation to explain the physical meaning of the pressure $p_{c}$ and to achieve the expression of the curve $p=\pi(\theta)$. Henceforth, we restrict our attention to a uniform configuration, i.e., $\nabla \varphi=\mathbf{0}$. 
By taking Eqs. (10) $)_{2}$ and (26) into account, we obtain

$$
\begin{aligned}
\eta_{0}(\rho, \theta, \varphi)=\eta(\rho, \theta, \varphi, \mathbf{0})= & -\partial_{\theta} \psi(\rho, \theta, \varphi, \mathbf{0})=c_{P} \\
& \ln \theta-k[1-v g(\varphi)] \ln \frac{p(\rho, \theta, \varphi)}{p_{c}} .
\end{aligned}
$$

In particular, the entropy of the vapor and of the liquid are given, respectively, by

$$
\begin{aligned}
& \eta_{0}(\rho, \theta, 0)=c_{P} \ln \theta-k \ln \frac{p(\rho, \theta, 0)}{p_{c}}, \\
& \eta_{0}(\rho, \theta, 1)=c_{P} \ln \theta-k(1-v) \ln \frac{p(\rho, \theta, 1)}{p_{c}} .
\end{aligned}
$$

At a point $\left(\theta^{*}, \pi\left(\theta^{*}\right)\right)$, the entropy of the vapor is greater than the entropy of the liquid, thus, the difference

$$
\eta_{0}\left(\rho, \theta^{*}, 0\right)-\eta_{0}\left(\rho, \theta^{*}, 1\right)=-k v \ln \frac{\pi\left(\theta^{*}\right)}{p_{c}}
$$

will be positive. Therefore, the pressure $p^{*}=\pi\left(\theta^{*}\right)$ has to satisfy the condition

$$
p^{*}<p_{c},
$$

namely the value $p_{c}$ is a physical upper bound for the pressure $p^{*}$.

In the Appendix, we show the following result.

Theorem 6.1 Given the constitutive equations (20) and the Helmholtz free energy density (26), the vapor pressure curve is given by

$$
p^{*}=\pi\left(\theta^{*}\right)=p_{c} e^{-\frac{L}{k v \theta^{*}}} .
$$

Moreover, the Clausius-Clapeyron equation holds, namely

$$
\frac{d \pi}{d \theta^{*}}=\frac{L \rho_{0} \rho_{1}}{\theta^{*}\left(\rho_{1}-\rho_{0}\right)},
$$

where $\rho_{0}$ and $\rho_{1}$ denote the density of the vapor and of the liquid.

Equations (30) and (31) coincide respectively with Eqs. (98) and (94) in [9].

It is apparent that in the $(\theta, p)$ plane the curve $\pi$ is a monotone increasing function and it satisfies the condition

$$
\lim _{\theta^{*} \rightarrow+\infty} \pi\left(\theta^{*}\right)=p_{c}
$$


Therefore, there exists the inverse function $\tau=\pi^{-1}$, which is given by

$$
\theta^{*}=\tau\left(p^{*}\right)=-\frac{L}{k v\left(\ln p^{*}-\ln p_{c}\right)} .
$$

Notice that since we have assumed $v=$ const, the critical temperature $\theta_{c}=$ $\tau\left(p_{c}\right)$ (i.e., the temperature above which transition never occurs) does not have a finite value.

\section{Governing equations}

Let us consider non-uniform phase changes. In view of Eqs. (4), (12), and (26), we obtain

$$
\begin{aligned}
\boldsymbol{T}= & -p \boldsymbol{I}+\chi \boldsymbol{D}-\mu_{0} \rho \nabla \varphi \otimes \nabla \varphi, \\
\frac{\rho \psi}{\theta}= & \rho\left[c_{P}(1-\ln \theta)+\frac{L}{\theta} f(\varphi)\right]+\rho k[1-\nu g(\varphi)] \\
& \left(\ln \frac{\rho k \theta[1-\nu g(\varphi)]}{p_{c}}-1\right)+\frac{\mu_{0}}{2} \rho|\nabla \varphi|^{2} .
\end{aligned}
$$

Accordingly, by substituting into Eqs. (2) and (5), the kinetic equations governing the evolution of $\rho, \boldsymbol{v}, \theta, \varphi$ read

$$
\begin{aligned}
& \dot{\rho}+\rho \nabla \cdot \boldsymbol{v}=0, \\
& \rho \dot{\boldsymbol{v}}=-\nabla p+\nabla \cdot(\chi \boldsymbol{D})-\mu_{0} \nabla \cdot(\rho \nabla \varphi \otimes \nabla \varphi)+\rho \boldsymbol{b}, \\
& \rho c(\varphi) \dot{\theta}+\left[\rho c^{\prime}(\varphi) \theta+L f^{\prime}(\varphi)\right] \dot{\varphi} \\
& \quad=-p \nabla \cdot \boldsymbol{v}+\chi \boldsymbol{D} \cdot \boldsymbol{D}-\mu_{0} \rho(\nabla \varphi \otimes \nabla \varphi) \cdot \boldsymbol{D}+\kappa \triangle \theta+\rho r, \\
& \dot{\varphi}=-\beta(\rho, \theta)\left[f^{\prime}(\varphi)+u g^{\prime}(\varphi)\right]+\lambda \mu_{0} \nabla \rho \cdot \nabla \varphi+\lambda \mu_{0} \rho \triangle \varphi,
\end{aligned}
$$

where $c(\varphi)$ is given in Eq. (21) and $\beta(\rho, \theta), u(p, \theta)$ are defined as

$$
\beta(\rho, \theta)=\lambda \rho \frac{L}{\theta}, \quad u(p, \theta)=-\frac{k \nu \theta}{L} \ln \frac{p}{p_{c}} .
$$

We append to Eq. (34) the boundary conditions

$$
\begin{aligned}
& \left.\nabla \theta \cdot \boldsymbol{n}\right|_{\partial \Omega}=0,\left.\quad \nabla \varphi \cdot \boldsymbol{n}\right|_{\partial \Omega}=0,\left.\quad \boldsymbol{v}\right|_{\partial \Omega}=\mathbf{0}, \\
& \rho(\boldsymbol{x}, 0)=\rho_{0}(\boldsymbol{x}), \quad \theta(\boldsymbol{x}, 0)=\theta_{0}(\boldsymbol{x}), \\
& \varphi(\boldsymbol{x}, 0)=\varphi_{0}(\boldsymbol{x}), \quad \boldsymbol{v}(\boldsymbol{x}, 0)=\boldsymbol{v}_{0}(\boldsymbol{x}) .
\end{aligned}
$$


In spite of their complexity, it is worth noting that this boundary value problem leads to a simple energy relation. Indeed, we multiply Eq. (2) $)_{2}$ by $\boldsymbol{v}$ and we add the resulting equation with Eq. (2) $)_{3}$, thus obtaining

$$
\frac{1}{2} \rho \frac{d}{d t}|\boldsymbol{v}|^{2}+\rho \dot{\varepsilon}=\nabla \cdot(\boldsymbol{T} \boldsymbol{v})+\kappa \Delta \theta+\rho \boldsymbol{b} \cdot \boldsymbol{v}+\rho r .
$$

We assume that the external forces are conservative, namely $\boldsymbol{b}=-\nabla \mathcal{V}$. In view of divergence theorem, continuity equation $(2)_{1}$, and boundary conditions (36), an integration over $\Omega$ leads to

$$
\begin{aligned}
\frac{d}{d t} \int_{\Omega}\left(\frac{1}{2} \rho|\boldsymbol{v}|^{2}+\rho \varepsilon+\rho \mathcal{V}\right) d v & = \\
& \int_{\partial \Omega}(\boldsymbol{T} \boldsymbol{v}+\kappa \nabla \theta) \cdot \boldsymbol{n} d a+\int_{\Omega} \rho r d v=\int_{\Omega} \rho r d v .
\end{aligned}
$$

\subsection{Phase transition at constant pressure}

We restrict our attention to phase transitions induced by the temperature and assume that the pressure $p$ is constant, $p=p^{*}$. In this assumption, $\theta^{*}=$ $\tau\left(p^{*}\right)$ is the so-called transition temperature at constant pressure (see [21]). Definition (35) 2 and Eq. (30) lead to

$$
u\left(p^{*}, \theta\right)=-\frac{k v \theta}{L} \ln \frac{p^{*}}{p_{c}}=\frac{\theta}{\theta^{*}} .
$$

Substitution of Eqs. (35) 1 and (38) into Eq. (34) 4 yields the evolution equation

$$
\begin{aligned}
\dot{\varphi} & =-\lambda \rho L\left[\frac{1}{\theta} f^{\prime}(\varphi)+\frac{1}{\theta^{*}} g^{\prime}(\varphi)\right]+\lambda \mu_{0} \nabla \rho \cdot \nabla \varphi+\lambda \mu_{0} \rho \triangle \varphi \\
& =-\lambda \rho L\left[\left(\frac{1}{\theta}-\frac{1}{\theta^{*}}\right) f^{\prime}(\varphi)+\frac{1}{\theta^{*}} \mathcal{W}^{\prime}(\varphi)\right]+\lambda \mu_{0} \nabla \rho \cdot \nabla \varphi+\lambda \mu_{0} \rho \triangle \varphi,
\end{aligned}
$$

where

$$
\mathcal{W}(\varphi)=f(\varphi)+g(\varphi) .
$$

As pointed out in the introduction, most phase-field models in the literature work at constant pressure. Thus, in the following, we establish a connection between our model and previous classical results concerning solid-liquid transitions obtained in [7] and [8]. To this end, we assume hereafter

$$
\rho=\text { const }=1, \quad \lambda=\text { const. }
$$

As a consequence, $\nabla \rho=\mathbf{0}$. 
First, we show that the "simpler model" proposed in Section 6.1 of [7] can be deduced from Eq. (39) as a particular case. More precisely, we take the expression of the free energy (6.14) in [7] into account, namely

$$
\bar{F}(\theta, \varphi, \nabla \varphi)=\theta\left[c \ln \frac{\theta^{*}}{\theta}+b(\varphi) \frac{\theta^{*}-\theta}{\theta^{*}}+\mathcal{G}(\varphi)+\frac{\alpha}{2}|\nabla \varphi|^{2}\right]+\text { const },
$$

and we recast $b$ and $\mathcal{G}$ so that $\varphi=0$ and $\varphi=1$ are the local minima of $\mathcal{G}$, that is

$$
b(\varphi)=\frac{2}{3} b_{0} \varphi^{3}(3 \varphi-4), \quad \mathcal{G}(\varphi)=4 b_{0} \varphi^{2}(\varphi-1)^{2}, \quad b_{0}>0 .
$$

Then, by letting

$$
\begin{array}{cc}
\lambda L=\frac{b_{0} \theta^{*}}{3 v}, & \lambda \mu_{0}=\frac{\alpha b_{0}}{v}, \\
f(\varphi)=\frac{3}{2 b_{0}} b(\varphi), & \mathcal{W}(\varphi)=\frac{3}{2 b_{0}} \mathcal{G}(\varphi),
\end{array}
$$

in Eq. (39), we recover the evolution equation (6.18) of [7].

Finally, we show that the model proposed in [8] can be obtained from Eq. (39) by means of a linearization with respect to $\theta$ in a neighborhood of $\theta^{*}$. To this end, we approximate $1 / \theta$ in the form

$$
\frac{1}{\theta} \approx \frac{1}{\theta^{*}}+\frac{1}{\left(\theta^{*}\right)^{2}}\left(\theta^{*}-\theta\right)
$$

thus obtaining

$$
\dot{\varphi}=-\frac{\lambda L}{\theta^{*}}\left[\frac{\theta^{*}-\theta}{\theta^{*}} f^{\prime}(\varphi)+\mathcal{W}^{\prime}(\varphi)\right]+\lambda \mu_{0} \triangle \varphi .
$$

It is worth noting that if we assume for $f$ and $\mathcal{W}$ the expressions given in Eq. (41), then the phase evolution is ruled by

$$
\dot{\varphi}=-\frac{12 \lambda L}{\theta^{*}} \varphi(\varphi-1)\left(\frac{\theta}{\theta^{*}} \varphi+\varphi-1\right)+\lambda \mu_{0} \triangle \varphi .
$$

Unfortunately, $\varphi$ does not satisfy the same evolution equation as [8]. If we want to recover exactly the evolution equation proposed in [8], we have to choose different expressions for $f$ and $\mathcal{W}$. In particular, we suppose that

$$
f(\varphi)=-3 \varphi^{4}+8 \varphi^{3}-6 \varphi^{2}, \quad \mathcal{W}(\varphi)=6 \varphi^{2}(\varphi-1)^{2} .
$$


Thus, by letting

$$
\frac{\lambda L}{\theta^{*}}=\frac{1}{\gamma}
$$

we obtain the evolution equation

$$
\dot{\varphi}=-\frac{1}{\gamma} \varphi(\varphi-1)\left[(\varphi-1) \frac{\theta}{\theta^{*}}+\varphi\right]+\lambda \mu_{0} \triangle \varphi,
$$

which coincides with $[8$, formula (22)].

\subsection{Phase transition at constant temperature}

Now we focus our attention on phase transitions induced by the pressure and we keep the temperature at the constant value $\theta=\theta^{*}$. In this case, $p^{*}=\pi\left(\theta^{*}\right)$ will be called transition pressure at constant temperature. From Eqs. (32) and $(35)_{2}$, we have

$$
u\left(p, \theta^{*}\right)=-\frac{k v \theta^{*}}{L} \ln \frac{p}{p_{c}}=\frac{\ln p-\ln p_{c}}{\ln p^{*}-\ln p_{c}} .
$$

By substituting this expression into Eq. (34) 4 , we deduce the evolution equation for $\varphi$

$$
\dot{\varphi}=-\beta\left(\rho, \theta^{*}\right)\left[f^{\prime}(\varphi)+\frac{\ln p-\ln p_{c}}{\ln p^{*}-\ln p_{c}} g^{\prime}(\varphi)\right]+\lambda \mu_{0} \nabla \rho \cdot \nabla \varphi+\lambda \mu_{0} \rho \triangle \varphi,
$$

where $f, g$ are as in Eq. (41), for instance.

In the literature, few papers deal with phase-field models working at constant temperature. So a comparison with previous classical results is hard to perform. In order to simplify our model, we approximate $\ln p$ in the neighborhood of $p^{*}$ by means of

$$
\ln p \approx \ln p^{*}+\frac{1}{p^{*}}\left(p-p^{*}\right) .
$$

Accordingly, $u$ is given by

$$
u=1+\xi\left(p-p^{*}\right),
$$

where

$$
\xi=\frac{1}{p^{*}\left(\ln p^{*}-\ln p_{c}\right)} .
$$


If this is the case, the evolution equation of $\varphi$ becomes

$$
\begin{aligned}
\dot{\varphi} & =-\frac{\lambda L \rho}{\theta^{*}}\left[f^{\prime}(\varphi)+\left[1+\xi\left(p-p^{*}\right)\right] g^{\prime}(\varphi)\right]+\lambda \mu_{0} \nabla \rho \cdot \nabla \varphi+\lambda \mu_{0} \rho \triangle \varphi \\
& =-\frac{\lambda L \rho}{\theta^{*}}\left[\xi\left(p-p^{*}\right) g^{\prime}(\varphi)+\mathcal{W}^{\prime}(\varphi)\right]+\lambda \mu_{0} \nabla \rho \cdot \nabla \varphi+\lambda \mu_{0} \rho \triangle \varphi .
\end{aligned}
$$

\subsection{Linearized governing equation}

As suggested by previous examples, we perform here a simultaneous linearization with respect to $\theta$ and $p$ in a neighborhood of a given transition point $\left(p^{*}, \theta^{*}\right), p^{*}=\pi\left(\theta^{*}\right)$. To this end, we assume that Eqs. (42) and (43) hold. From Eq. (35) $)_{2}$ we obtain

$$
\frac{u}{\theta}=-\frac{k v}{L}\left(\ln p-\ln p_{c}\right) \approx-\frac{k v}{L}\left(\ln p^{*}-\ln p_{c}\right)-\frac{k v}{L} \frac{p-p^{*}}{p^{*}}
$$

Moreover, since $p^{*}=\pi\left(\theta^{*}\right)$, relation (30) yields

$$
\frac{u}{\theta} \approx \frac{1}{\theta^{*}}-\frac{k v}{L} \frac{p-p^{*}}{p^{*}}
$$

Accordingly, substitution of Eqs. (43) and (46) into Eq. (34) 4 leads to the evolution equation for the phase field

$$
\begin{aligned}
\dot{\varphi}=-\frac{\lambda L}{\theta^{*}} \rho\left[\frac{\theta^{*}-\theta}{\theta^{*}} f^{\prime}(\varphi)-\frac{k \nu \theta^{*}}{L}\right. & \left.\frac{p^{*}-p}{p^{*}} g^{\prime}(\varphi)+\mathcal{W}^{\prime}(\varphi)\right] \\
& +\lambda \mu_{0} \nabla \rho \cdot \nabla \varphi+\lambda \mu_{0} \rho \triangle \varphi .
\end{aligned}
$$

It is worth noting that if we keep the pressure $p$ constant, $p=p^{*}$, Eq. (47) has the same form as Eq. (39), whereas, if we suppose $\theta=\theta^{*}=$ const, by taking Eq. (32) into account, we recover Eq. (45).

\section{Conclusions}

In this paper, we have proposed a thermodynamically consistent phase-field model able to describe the transition between liquid and vapor. In this approach, the interfaces between the two pure phases are regarded as regions of finite width having a gradual variation of different physical quantities. In order to distinguish one phase from the other, we have introduced an auxiliary variable $\varphi$ whose evolution is assumed to be governed by the equation 
$\dot{\varphi}=\Phi(\Sigma)$. Since $\Delta \varphi$ is expected to enter the explicit expression of this equation, we are forced to consider a non-local thermodynamic framework, where first and second gradients of all the unknown fields, $\rho, \theta, \varphi$ and $\boldsymbol{D}^{\circ}$, are involved in $\Sigma$. Unlike classical phase-field models, the kinetic equation for the phase variable is obtained as a consequence of thermodynamic restrictions and it depends only on the rescaled free energy $\psi / \theta$. Since the shape of $\psi$ is related to the expressions of the pressure and the internal energy, first we have assumed simplified constitutive equations for $p$ and $\varepsilon$ in the pure phases. Later, we have extended them to the whole domain, having in mind that during the transition pressure and temperature are constant. While it is apparent and experimentally verified which are the constitutive equations in the pure phases, it is not evident which are the constitutive equations in the transition layer. In a more realistic modeling we should have chosen a different constitutive equation for the pressure in the liquid phase and a non-linear dependence on the temperature for the internal energy in agreement with the principle of equipresence in rational thermodynamics. On the other hand, such choices lead to more complex models, which are less tractable from a mathematical point of view.

\section{Appendix}

\section{Proof of proposition 3.2}

The free energy density $\psi$ is a function of $\Sigma$. Thus, by evaluating the time derivative of $\psi$ and substituting into Eq. (9), we obtain

$$
\begin{aligned}
& -\rho\left[\partial_{\rho} \psi \dot{\rho}+\left(\partial_{\theta} \psi+\eta\right) \dot{\theta}+\partial_{\varphi} \psi \dot{\varphi}+\partial_{\boldsymbol{D}^{\circ}} \psi \cdot \dot{\boldsymbol{D}}^{\circ}\right. \\
& +\partial_{\nabla \rho} \psi \cdot \dot{\bar{\nabla} \rho}+\partial_{\nabla \theta} \psi \cdot \dot{\bar{\nabla} \theta} \\
& +\partial_{\nabla \varphi} \psi \cdot \dot{\bar{\nabla} \varphi}+\partial \nabla \boldsymbol{D}^{\circ} \psi \cdot \dot{\bar{\nabla} \boldsymbol{D}^{\circ}}+\partial_{\nabla \nabla \rho} \psi \dot{\nabla \nabla} \rho \\
& \left.+\partial_{\nabla \nabla \theta} \psi \dot{\nabla \nabla} \overline{\nabla \theta}+\partial_{\nabla \nabla \varphi} \psi \dot{\nabla \nabla} \dot{\nabla}\right] \\
& +\boldsymbol{T} \cdot \boldsymbol{D}-\frac{1}{\theta} \boldsymbol{q} \cdot \nabla \theta+\theta \nabla \cdot \boldsymbol{k} \geq 0 .
\end{aligned}
$$

As shown in [7], on account of Eq. (5), one can easily check

$$
\begin{aligned}
& \dot{\nabla \varphi}=\nabla \dot{\varphi}-\boldsymbol{L}^{T} \nabla \varphi=\nabla \Phi-\boldsymbol{L}^{T} \nabla \varphi, \\
& \partial_{\nabla \varphi} \psi \cdot \boldsymbol{L}^{T} \nabla \varphi=(\nabla \varphi \otimes \partial \nabla \varphi \psi) \cdot \boldsymbol{L},
\end{aligned}
$$


where the symbol ${ }^{T}$ stands for the transpose of a tensor. Accordingly, substitution of Eqs. (1), (3)-(5) into Eq. (48) leads to

$$
\begin{aligned}
& -\rho\left[\left(\partial_{\theta} \psi+\eta\right) \dot{\theta}+\partial_{\varphi} \psi \Phi+\partial_{\boldsymbol{D}^{\circ}} \psi \cdot \dot{\boldsymbol{D}}^{\circ}+\partial_{\nabla \rho} \psi \cdot \dot{\bar{\nabla} \rho}\right. \\
& +\partial_{\nabla \theta} \psi \cdot \dot{\nabla} \theta+\partial_{\nabla \varphi} \psi \cdot \nabla \Phi
\end{aligned}
$$

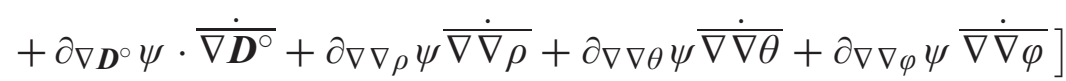

$$
\begin{aligned}
& +\left(\frac{p}{\rho}-\rho \partial_{\rho} \psi\right) \dot{\rho} \\
& +[\boldsymbol{S}+\rho \operatorname{sym}(\nabla \varphi \otimes \partial \nabla \varphi \psi)] \cdot \boldsymbol{D}+\rho \operatorname{skw}(\nabla \varphi \otimes \partial \nabla \varphi \psi) \cdot \boldsymbol{\Omega} \\
& -\frac{1}{\theta} \boldsymbol{q} \cdot \nabla \theta+\theta \nabla \cdot \boldsymbol{k} \geq 0 .
\end{aligned}
$$

By virtue of the arbitrariness of $\dot{\rho}, \dot{\theta}, \dot{\boldsymbol{D}}^{\circ}, \dot{\bar{\nabla} \rho}, \dot{\nabla} \theta, \nabla \dot{\boldsymbol{D}}^{\circ}, \frac{\bar{\nabla} \bar{\nabla} \rho}{\bar{\nabla} \dot{\nabla} \theta}, \mathbf{\Omega}$ for any fixed value of $\rho, \theta, \boldsymbol{D}^{\circ}, \nabla \rho, \nabla \theta, \nabla \dot{\boldsymbol{D}}^{\circ}, \nabla \nabla \rho, \nabla \nabla \theta$ at $(\boldsymbol{x}, t) \in \Omega$, we obtain

$$
\begin{aligned}
& \eta=-\partial_{\theta} \psi, \quad \partial \nabla \rho \psi \partial \nabla \nabla \psi=\partial_{\boldsymbol{D}^{\circ} \psi=\mathbf{0},} \\
& \partial_{\rho} \psi=\frac{p}{\rho^{2}}, \quad \partial \nabla \boldsymbol{D}^{\circ} \psi=\partial \nabla \nabla \rho=\partial_{\nabla \nabla \theta} \psi=\mathbf{0}, \\
& \operatorname{skw}(\nabla \varphi \otimes \partial \nabla \varphi \psi)=\mathbf{0} .
\end{aligned}
$$

Furthermore, the non-trivial dependence of $\Phi$ on $\nabla \varphi$ guarantees that even $\overline{\nabla \nabla \varphi}$ is arbitrary. This can be proved by standard arguments [7] and implies the condition

$$
\partial \nabla \nabla \varphi \psi=\mathbf{0} .
$$

As a consequence, Eq. (48) reduces to

$$
\begin{aligned}
-\rho \partial_{\varphi} \psi \Phi-\rho \partial \nabla \varphi \psi \cdot \nabla \Phi+(\boldsymbol{S}+\rho \nabla \varphi \otimes \partial \nabla \varphi \psi) \cdot \boldsymbol{D} \\
\\
\quad-\frac{1}{\theta} \boldsymbol{q} \cdot \nabla \theta+\theta \nabla \cdot \boldsymbol{k} \geq 0 .
\end{aligned}
$$

Since both $\boldsymbol{k}$ and $\Phi$ are assumed to be functions of $\Sigma$, by evaluating $\nabla \cdot \boldsymbol{k}$ and $\nabla \Phi$, we obtain

$$
\begin{array}{r}
-\rho \partial_{\varphi} \psi \Phi+\left(\theta \partial_{\rho} \boldsymbol{k}-\rho \partial_{\nabla \varphi} \psi \partial_{\rho} \Phi\right) \cdot \nabla \rho \\
+\left(\theta \partial_{\theta} \boldsymbol{k}-\frac{1}{\theta} \boldsymbol{q}-\rho \partial_{\nabla \varphi} \psi \partial_{\theta} \Phi\right) \cdot \nabla \theta
\end{array}
$$




$$
\begin{aligned}
& +\left(\theta \partial_{\varphi} \boldsymbol{k}-\rho \partial_{\nabla \varphi} \psi \partial_{\varphi} \Phi\right) \cdot \nabla \varphi+\left(\theta \partial_{\boldsymbol{D}^{\circ} \boldsymbol{k}}-\rho \partial \partial_{\varphi} \psi \otimes \partial \partial^{\circ} \Phi\right) \cdot \nabla \boldsymbol{D}^{\circ} \\
& +\left(\theta \partial \partial_{\rho} \boldsymbol{k}-\rho \partial_{\nabla \varphi} \psi \otimes \partial \partial_{\nabla} \Phi\right) \cdot \nabla \nabla \rho \\
& +\left(\theta \partial_{\nabla \theta} \boldsymbol{k}-\rho \partial_{\nabla \varphi} \psi \otimes \partial \nabla \theta \Phi\right) \cdot \nabla \nabla \theta \\
& +\left(\theta \partial \nabla_{\varphi} \boldsymbol{k}-\rho \partial \nabla_{\varphi} \psi \otimes \partial \nabla_{\varphi} \Phi\right) \cdot \nabla \nabla \varphi \\
& +\left(\theta \partial \nabla \boldsymbol{D}^{\circ} \boldsymbol{k}-\rho \partial_{\nabla \varphi} \psi \otimes \partial \nabla \boldsymbol{D}^{\circ} \Phi\right) \cdot \nabla \nabla \boldsymbol{D}^{\circ} \\
& +\left(\theta \partial_{\nabla \nabla \rho} \boldsymbol{k}-\rho \partial_{\nabla \varphi} \psi \otimes \partial \partial_{\nabla} \rho \Phi\right) \cdot \nabla \nabla \nabla \rho \\
& +\left(\theta \partial_{\nabla \nabla \theta} \boldsymbol{k}-\rho \partial_{\nabla \varphi} \psi \otimes \partial_{\nabla \nabla \theta} \Phi\right) \cdot \nabla \nabla \nabla \theta \\
& +(\theta \partial \nabla \nabla \varphi)-\rho \partial \nabla \varphi \psi \otimes \partial \nabla \nabla \varphi \Phi) \cdot \nabla \nabla \nabla \varphi \\
& +\left(\boldsymbol{S}+\rho \nabla \varphi \otimes \partial_{\nabla \varphi} \psi\right) \cdot \boldsymbol{D} \geq 0 .
\end{aligned}
$$

The arbitrariness of the derivatives $\nabla \nabla \boldsymbol{D}^{\circ}, \nabla \nabla \nabla \rho, \nabla \nabla \nabla \theta, \nabla \nabla \nabla \varphi$, which are all independent of $\Sigma$, and the independence of $\psi$ on $\nabla \boldsymbol{D}^{\circ}, \nabla \nabla \rho, \nabla \nabla \theta$, $\nabla \nabla \varphi$ lead to

$$
\begin{array}{ll}
\partial \nabla \boldsymbol{D}^{\circ}\left(\theta \boldsymbol{k}-\rho \partial \nabla_{\varphi} \psi \Phi\right)=\mathbf{0}, & \partial \nabla_{\nabla}\left(\theta \boldsymbol{k}-\rho \partial \nabla_{\varphi} \psi \Phi\right)=\mathbf{0}, \\
\partial_{\nabla \nabla \theta}\left(\theta \boldsymbol{k}-\rho \partial_{\nabla \varphi} \psi \Phi\right)=\mathbf{0}, & \partial \nabla_{\nabla \varphi}\left(\theta \boldsymbol{k}-\rho \partial_{\nabla \varphi} \psi \Phi\right)=\mathbf{0} .
\end{array}
$$

Inequality (50) cannot be further reduced by means of standard arguments. Thus, we adopt a different approach based on Assumption 3.1. Firstly we show that the entropy extra flux is given by

$$
\boldsymbol{k}(\Sigma)=\frac{1}{\theta} \rho \partial \nabla \varphi \psi \dot{\varphi}=\frac{1}{\theta} \rho \partial \nabla_{\varphi} \psi \Phi,
$$

namely, we show that $\boldsymbol{k}$ is uniquely determined. Indeed, from Eq. (51) it follows that

$$
\theta \boldsymbol{k}-\rho \partial \nabla \varphi \psi \Phi
$$

is independent of $\nabla \boldsymbol{D}^{\circ}, \nabla \nabla \rho, \nabla \nabla \theta, \nabla \nabla \varphi$. Hence, the entropy extra flux $\boldsymbol{k}$ has to satisfy

$$
\theta \boldsymbol{k}(\Sigma)=\rho \partial_{\nabla \varphi} \psi \Phi(\Sigma)+\boldsymbol{h}\left(\rho, \theta, \varphi, \boldsymbol{D}^{\circ}, \nabla \rho, \nabla \theta, \nabla \varphi\right),
$$

where $\boldsymbol{h}$ is an arbitrary function of $\rho, \theta, \varphi, \boldsymbol{D}^{\circ}, \nabla \rho, \nabla \theta, \nabla \varphi$. Therefore, on account of Assumption 3.1, we deduce $\boldsymbol{h} \equiv \mathbf{0}$ and Eq. (52) holds.

Finally, substitution of Eq. (52) into Eq. (49) leads to

$$
-\rho \partial_{\varphi} \psi \Phi+(\boldsymbol{S}+\rho \nabla \varphi \otimes \partial \nabla \varphi \psi) \cdot \boldsymbol{D}-\frac{1}{\theta} \boldsymbol{q} \cdot \nabla \theta+\theta \nabla \cdot\left(\frac{\rho}{\theta} \partial \nabla \varphi \psi\right) \Phi \geq 0
$$

and Eq. (11) is proved. 
Proof of theorem 6.1 First, we prove Eq. (30). When the pair $(\theta, p)$ belongs to the curve separating the two phases, i.e., $p^{*}=\pi\left(\theta^{*}\right)$, vapor and liquid coexist. Let $\rho_{0}, \rho_{1}$ be the densities respectively of the vapor and the liquid at $\left(p^{*}, \theta^{*}\right)$. It is well known (see, e.g., [12]), that in such a condition $\gamma$ takes the same value at the two pure phases, namely

$$
\gamma\left(\rho_{0}, \theta^{*}, 0, \mathbf{0}\right)=\gamma_{0}\left(\rho_{1}, \theta^{*}, 1, \mathbf{0}\right) .
$$

Since during the transition pressure and temperature are constant, it is convenient to write the previous condition in the equivalent form

$$
\tilde{\gamma}_{0}\left(p^{*}, \theta^{*}, 0\right)=\tilde{\gamma}_{0}\left(p^{*}, \theta^{*}, 1\right),
$$

where, in view of Eq. (27),

$$
\begin{aligned}
\tilde{\gamma}_{0}(p, \theta, \varphi)=\tilde{\gamma}(p, \theta, \varphi, \mathbf{0}) \\
\quad=c_{P} \theta(1-\ln \theta)+L f(\varphi)+k \theta[1-v g(\varphi)] \ln \frac{p}{p_{c}} .
\end{aligned}
$$

By defining

$$
u(p, \theta)=-\frac{k v \theta}{L} \ln \frac{p}{p_{c}}
$$

$\tilde{\gamma}_{0}$ can be written as

$$
\tilde{\gamma}_{0}(p, \theta, \varphi)=c_{p} \theta(1-\ln \theta)+k \theta \ln \frac{p}{p_{c}}+L[f(\varphi)+u(p, \theta) g(\varphi)] .
$$

Therefore, condition (54) reads

$$
f(0)+u\left(p^{*}, \theta^{*}\right) g(0)=f(1)+u\left(p^{*}, \theta^{*}\right) g(1) .
$$

From relations (19), it follows that $u\left(p^{*}, \theta^{*}\right)=1$ on the vapor-pressure curve. Accordingly, by letting $u\left(\theta^{*}, \pi\left(\theta^{*}\right)\right)=1$ in Eq. (35), we find out the expression of the vapor-pressure curve, which is

$$
p^{*}=\pi\left(\theta^{*}\right)=p_{c} e^{-\frac{L}{k v \theta^{*}}} .
$$

Now we deduce the Clausius-Clapeyron equation. Let us consider Eq. (54) and differentiate it with respect to $\theta^{*}$, thus obtaining

$$
\begin{aligned}
\partial_{p^{*}} \tilde{\gamma}_{0}\left(p^{*}, \theta^{*}, 0\right) \frac{d \pi}{d \theta^{*}} & +\partial_{\theta^{*}} \tilde{\gamma}_{0}\left(p^{*}, \theta^{*}, 0\right) \\
& =\partial_{p^{*}} \tilde{\gamma}_{0}\left(p^{*}, \theta^{*}, 1\right) \frac{d \pi}{d \theta^{*}}+\partial_{\theta^{*}} \tilde{\gamma}_{0}\left(p^{*}, \theta^{*}, 1\right),
\end{aligned}
$$


where $p^{*}=\pi\left(\theta^{*}\right)$. We differentiate $\tilde{\gamma}_{0}$ given in Eq. (55) with respect to $p$ and $\theta$. A comparison with Eqs. (17) and (28) leads to

$$
\begin{aligned}
& \partial_{p} \tilde{\gamma}_{0}(p, \theta, \varphi)=\frac{k \theta[1-v g(\varphi)]}{p}=\frac{1}{\rho}, \\
& \partial_{\theta} \tilde{\gamma}_{0}(p, \theta, \varphi)=-c_{P} \ln \theta+k[1-v g(\varphi)] \ln \frac{p}{p_{c}}=-\tilde{\eta}_{0}(p, \theta, \varphi),
\end{aligned}
$$

with $\tilde{\eta}_{0}(p, \theta, \varphi)=\eta_{0}(\rho, \theta, \varphi)$. Therefore, substituting into Eq. (56), we obtain the Clausius-Clapeyron equation

$$
\frac{d p^{*}}{d \theta^{*}}=\frac{\tilde{\eta}_{0}\left(\pi\left(\theta^{*}\right), \theta^{*}, 0\right)-\tilde{\eta}_{0}\left(\pi\left(\theta^{*}\right), \theta^{*}, 1\right)}{\frac{1}{\rho_{0}}-\frac{1}{\rho_{1}}},
$$

according to well-known results on thermodynamics (see, e.g., [12]).

Since $u=1$ holds along the curve separating the two stable phases, from Eq. (29) we have

$$
\begin{aligned}
& \tilde{\eta}_{0}\left(\pi\left(\theta^{*}\right), \theta^{*}, 0\right)-\tilde{\eta}_{0}\left(\pi\left(\theta^{*}\right), \theta^{*}, 1\right) \\
& \quad=-k v \ln \frac{\pi\left(\theta^{*}\right)}{p_{c}}=L \frac{u\left(\pi\left(\theta^{*}\right), \theta^{*}\right)}{\theta^{*}}=\frac{L}{\theta^{*}},
\end{aligned}
$$

which guarantees that the difference of the entropies of the vapor and of the liquid is equal to $L / \theta^{*}$. Substitution into Eq. (57) leads to a simplified form of the Clausius-Clapeyron equation,

$$
\frac{d \pi}{d \theta^{*}}=\frac{L \rho_{0} \rho_{1}}{\theta^{*}\left(\rho_{1}-\rho_{0}\right)} .
$$

\section{Acknowledgment}

We are grateful to the referees for their valuable suggestions and comments. 


\section{References}

[1] Alt, H.W., Pawlow, I., A mathematical model of dynamics of non-isothermal phase separation, Phys. D, 59 (1992), 389-416.

[2] Alt, H.W., Pawlow, I., On the entropy principle of phase transition models with a conserved parameter, Adv. Math. Sci. Appl., 6 (1996), 291-376.

[3] Brokate, M., Sprekels, J., Hysteresis and Phase Transitions, Springer, New York, 1996.

[4] Caginalp, G., An analysis of a phase field model of a free boundary, Arch. Rational Mech. Anal., 92 (1986), 205-245.

[5] Coleman, B., Noll, W., The thermodynamics of elastic materials with heat conduction and viscosity, Arch. Rational Mech. Anal., 13 (1963), 167-178.

[6] Fabrizio, M., Ice-water and liquid-vapor phase transitions by a Ginzburg-Landau model, J. Math. Phys., 49 (2008), 102902.

[7] Fabrizio, M., Giorgi, C., Morro, A., A thermodynamic approach to non-isotermal phase-field evolution in continuum physics, Phys. D, 214 (2006), 144-156.

[8] Fabrizio, M., Giorgi, C., Morro, A., A continuum theory for first-order phase transitions based on the balance of the structure order, Math. Methods Appl. Sci., 31 (2008), 627-653.

[9] Fermi, E., Thermodynamics, Dover, New York, 1956.

[10] Fried, E., Gurtin, M.E., Continuum theory of thermally induced phase transitions based on an order parameter, Phys. D, 68 (1993), 326-343.

[11] Green, A.E., Laws, N., On a global entropy production inequality, Quart. J. Mech. Appl. Math., 25 (1972), 1-11.

[12] Greiner, W., Neise, L., Stöcker, H., Thermodynamics and Statistical Mechanics, Springer, Berlin, 1994.

[13] Karma, A., Rappel, W.J., Quantitative phase-field modeling of dendritic growth in two and three dimensions, Phys. Rev. E, 57 (1998), 4323-4349.

[14] Langer, J.S., Models of pattern formation in first-order phase transition, in: Directions in Condensed Matter Physics, 165-186, World Scientific, Singapore, 1986.

[15] Maugin, G.A., The Thermomechanics of Nonlinear Irreversible Behaviors. An Introduction, World Scientific, Singapore, 1999.

[16] Morro, A., Phase-field models for fluid mixtures, Math. Comput. Modelling, 45 (2007), 1042-1052.

[17] Morro, A., A phase-field approach to non-isothermal transitions, Math. Comput. Modelling, 48 (2008), 621-633.

[18] Müller, I., Thermodynamics. Pitman, Boston, 1985.

[19] Ode, M., Kim, S.G., Suzuki, T., Recent advances in the phase-field model for solidification, ISIJ Int., 41 (2001), 1076-1082.

[20] Penrose, O., Fife, P.C., Thermodynamically consistent models of phase-field type for the kinetics of phase transitions, Phys. D, 43 (1990), 44-62.

[21] Penrose, O., Fife, P.C., On the relation between the standard phase-field model and a "thermodynamically consistent" phase-field model, Phys. D, 69 (1993), 107-113.

[22] Singer-Loginova, I., Singer, H.M., The phase-field technique for modeling multiphase materials, Rep. Prog. Phys., 71 (2008), 106501-106532. 
[23] Smith, J., Hendrick, M., Van Ness, C., Abbott, M., Introduction to Chemical Engineering Thermodynamics, McGraw Hill, Boston, 2001.

[24] Visintin, A., Introduction to Stefan-type problems, in: C.M. Dafermos, M. Pokorny, editors, Handbook of Differential Equations: Evolutionary Equations, vol. 4. chapter 8, 377-484, North-Holland, Amsterdam, 2008.

[25] Yeh, C.S., Chen, K.C., On the continuum mechanical description for elastic typeII superconductors, Proc. R. Soc. Lond. Ser. A Math. Phys. Eng. Sci., 452 (1944) (1996), 139-149.

Paper received: 2009-01-28

Paper accepted: $\quad$ 2009-04-28 